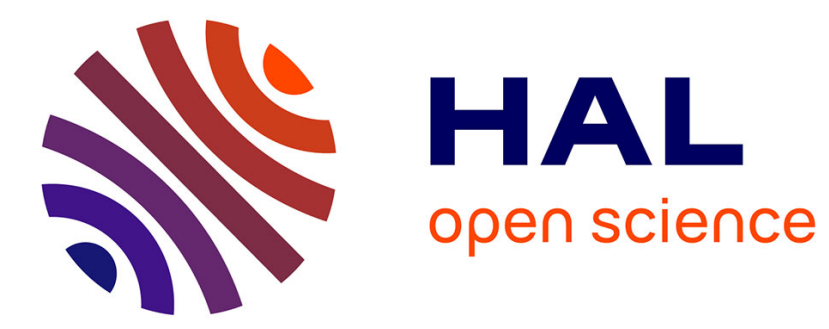

\title{
SEXUALITÉ ET COMMUNAUTÉ FAMILIALE, LE REGARD DE L'ANTHROPOLOGIE
}

\author{
Paul Rasse
}

\section{To cite this version:}

Paul Rasse. SEXUALITÉ ET COMMUNAUTÉ FAMILIALE, LE REGARD DE L'ANTHROPOLOGIE. Hermès, La Revue - Cognition, communication, politique, 2014, 69, pp.135-140. hal-03276697

\section{HAL Id: hal-03276697 \\ https://hal.science/hal-03276697}

Submitted on 15 Oct 2021

HAL is a multi-disciplinary open access archive for the deposit and dissemination of scientific research documents, whether they are published or not. The documents may come from teaching and research institutions in France or abroad, or from public or private research centers.
L'archive ouverte pluridisciplinaire HAL, est destinée au dépôt et à la diffusion de documents scientifiques de niveau recherche, publiés ou non, émanant des établissements d'enseignement et de recherche français ou étrangers, des laboratoires publics ou privés. 


\title{
SEXUALITÉ ET COMMUNAUTÉ FAMILIALE, LE REGARD DE L'ANTHROPOLOGIE
}

\section{$\underline{\text { Paul Rasse }}$}

\author{
C.N.R.S. Editions | « Hermès, La Revue »
}

$2014 / 2 \mathrm{n}^{\circ} 69 \mid$ pages 135 à 140

ISSN 0767-9513

ISBN 9782271082176

\section{Article disponible en ligne à l'adresse :}

https://www.cairn.info/revue-hermes-la-revue-2014-2-page-135.htm

Distribution électronique Cairn.info pour C.N.R.S. Editions.

(C) C.N.R.S. Editions. Tous droits réservés pour tous pays.

La reproduction ou représentation de cet article, notamment par photocopie, n'est autorisée que dans les limites des conditions générales d'utilisation du site ou, le cas échéant, des conditions générales de la licence souscrite par votre établissement. Toute autre reproduction ou représentation, en tout ou partie, sous quelque forme et de quelque manière que ce soit, est interdite sauf accord préalable et écrit de l'éditeur, en dehors des cas prévus par la législation en vigueur en France. Il est précisé que son stockage dans une base de données est également interdit. 


\section{Paul Rasse}

Université Nice-Sophia Antipolis

\section{Sexualité et communauté familiale, le regard de l'anthropologie}

Les anthropologues et les psychologues en conviennent, la sexualité est d'abord un fait de culture. La libido ne désigne pas d'objet de satisfaction et les voies pour l'assouvir sont nombreuses, diversifiées, variant profondément d'une société à l'autre. Cependant, elles ont toutes comme caractéristique commune d'être socialement très codifiées, comme si les sociétés s'en étaient depuis toujours méfiées, considérant la sexualité comme une source d'énergie incandescente. Aussi, depuis la nuit des temps, ont-elles confié à la famille le soin de l'enserrer pour la sublimer et en capter la puissance en fonction de ses propres besoins: la nécessité absolue de se reproduire et d'assurer les conditions indispensables à son existence.

La famille semble l'unité de base communautaire, qui offre suffisamment de solidarité intergénérationnelle pour abriter la vie, la maintenir et la transmettre de génération en génération. Les anthropologues la définissent comme un ensemble d'hommes, de femmes et d'enfants, unis par des liens légaux, par des droits, des obligations et des intérêts de nature économique ou religieuse, par un réseau précis de règles et d'interdits sexuels, auxquels s'ajoute un ensemble variable de sentiments tels que l'amour, l'affec- tion, le respect ou la crainte. Progressivement, la famille nucléaire contemporaine s'est imposée à nous et sur la planète tout entière comme étant la seule normale, alors qu'elle n'en est qu'une des formes possibles. Comme LéviStrauss (1956) le pressentait, tant qu'elle avait une grande valeur fonctionnelle, la famille s'actualisait bien au-dessus de ce niveau, jusqu'à former une communauté très étendue pouvant compter plusieurs centaines de membres; lorsqu'elle offre une faible valeur fonctionnelle, elle tend à disparaître en dessous du niveau de type conjugal, comme en témoigne la progression des familles monoparentales réduites au couple mère/enfant.

Nous rappellerons comment, en créant artificiellement de la différence par des faits de culture, et non pas de nature, les sociétés premières ont inventé des façons de dominer la sexualité et de générer du lien social solide, indéfectible, pour tisser les communautés. Puis nous nous demanderons en quoi cela nous permet de réfléchir à la famille contemporaine, générée sur la base du désir, cimentée par l'amour romantique et une sexualité épanouie, mais apparemment soumise à une obligation de fidélité inconditionnelle (Rasse, 2006). 


\section{Interdits et injonctions culturelles pour réglementer la sexualité des premières communautés}

Certaines théories défendent qu'à la différence de la plupart des animaux, où les femelles n'acceptent l'accouplement que lorsqu'elles sont fertiles, les femmes peuvent avoir et souhaiter des relations sexuelles à tout moment. Cela aurait contribué à la naissance de relations affectives durables, expliquant le développement de la famille monogamique, composée du père légitime, de la mère et de leurs enfants. Or, tout contredit cette vision romantique d'une communauté familiale unie par le désir libidinal et l'affection. Et si cette dernière en est peut-être l'aboutissement, comme la sexualité en ferait l'énergie, l'une et l'autre ne sont pas bien convaincantes quand il s'agit d'expliquer l'universalité et la stabilité de la famille.

Claude Lévi-Strauss (1981) s'est efforcé de montrer qu'en dépit de la diversité des formes apparentes de la famille, dont le recensement et le décryptage ont tellement passionné et occupé les anthropologues, quelques grands principes en forment la structure universelle ( $c f$. Deliège, 1996). En reprenant ses travaux, Françoise Héritier (1996) en distingue trois - " 1 . la répartition sexuelle des tâches, 2. la prohibition de l'inceste, 3. l'instauration de formes reconnues d'union matrimoniale» - qui constituent, dit-elle, les trois piliers de la famille et de la société. Les deux premiers renvoient à des interdictions archaïques, quasi universelles, fondatrices de l'échange et donc de la vie sociale, tandis que la troisième catégorie concerne plutôt une série d'injonctions destinées à organiser le vivre ensemble, car partout des règles d'ordre culturel, faites d'interdits et d'injonctions, de droits et de devoirs, assurent la cohésion interne de la famille, son existence matérielle et sa perpétuation de génération en génération.

Pierre Clastres (2001), par exemple, montre bien que les sociétés primitives distinguent soigneusement certaines tâches, strictement réservées aux hommes - la chasse notamment - d'autres qui ne peuvent être exécutées que par des femmes - en général liées à la vie domestique. Dès lors, en conclut Claude Lévi-Strauss (1971), il faut obligatoirement pour assumer l'ensemble des travaux nécessaires à l'existence de la communauté qu'hommes et femmes vivent ensemble, "cette division sexuelle du travail n'est pas autre chose qu'un simple moyen d'instituer un état de dépendance réciproque entre les sexes». Autrement dit, pour fonder une famille et lui permettre de durer, les hommes premiers ont inventé une forme d'attraction infiniment plus forte que celle de la sexualité, du désir et des sentiments, qui redouble et inscrit les différences biologiques dans la culture, de façon à rendre le couple «mari et femme» irréductiblement dépendant, alors que la sexualité dure ce qu'elle dure et que le sentiment amoureux qui en découle est sujet à bien des évolutions et des renversements.

Une autre règle, universelle cette fois, celle de l'inceste, a elle aussi fait couler beaucoup d'encre. Robin Fox (1972) y voit l'interdiction de la famille à se refermer sur elle-même et l'obligation de nouer un réseau d'alliances pacifiques durables, un système d'échange matrimonial entre les clans: le connubium. Et Claude Lévi-Strauss (1981) d'expliquer que «la prohibition de l'inceste est moins une règle qui interdit d'épouser mère, sœur ou fille que la règle qui oblige à donner mère, sœur, ou fille à autrui». C'est la règle du don par excellence. «De même que le principe de la division du travail établit une indépendance mutuelle entre les sexes, les emmenant par là à se perpétuer et à fonder une famille, la prohibition de l'inceste institue une dépendance mutuelle entre les familles les forçant à engendrer de nouvelles familles en vue de se perpétuer», conclut-il (Lévi-Strauss, 1971). La culture, la raison, l'intelligence, l'emportent sur les pulsions archaïques. Claude Lévi-Strauss y voit l'acte fondateur de l'humanité.

Dès lors, une autre série de règles, devient essentielle, celles du lignage. Elles visent à inscrire chaque famille 
dans la durée en gardant la trace des ancêtres et en assignant les conditions dans lesquelles doit se faire le choix du partenaire sexuel pour produire la génération suivante, à laquelle sera transmis le patrimoine de la famille.

Dans les sociétés patrilinéaires (l'un des deux grands versants où se distribuent les formes possibles de la famille), les relations sexuelles entre parents sont le noyau du groupe. Les femmes légitimes habitent avec leur mari, le règlement de la sexualité, son contrôle et les sanctions qui accompagnent les écarts sont extrêmement rigoureux. La soumission des femmes à l'autorité des hommes est plus forte. Il est en effet indispensable à la cohésion du groupe et de la famille, mais surtout à la poursuite de la lignée, à la transmission du nom et du patrimoine, de garantir que le père est bien le géniteur. Aussi faut-il tenir la sexualité enserrée dans une gangue d'acier et surveiller la famille comme on le ferait d'une chaudière que le désir et le sentiment amoureux sont toujours prêts à faire exploser.

Inversement, les sociétés matriarcales sont très libérales; comme les hommes assurent leur filiation par leurs sœurs, ils sont, une fois pour toutes, débarrassés de l'inquiétude généalogique tenace qui taraude les sociétés patrilinéaires: savoir s'ils sont bien le père. Le couple biologique (père géniteur/mère de l'enfant) est différent du couple parental légitime composé du frère et de la sœur; comme si, pour fonder durablement la famille et assurer sa perpétuation, les sociétés matrilinéaires s'étaient méfiées du sentiment amoureux ayant sa source dans la libido et l'accouplement, en lui préférant les sentiments maternels et fraternels. De fait, la sexualité est disjointe de la reproduction de la famille, puisque les femmes ont un ou plusieurs amants qui les fécondent, avec lesquels elles ont des relations épisodiques ou suivies, avec lesquels elles peuvent vivre ou non; peu importe en définitive, puisque de toute façon l'enfant appartient au clan de la mère, c'est-à-dire, en fait, à celui du frère de la mère, que l'enfant ira rejoindre au plus tard à la puberté. C'est le cas des Trobriandais de Mélanésie décrits par Bronisław Malinowski (1930).
Ceux-ci pensent que la mère est autofécondée; ils n'identifient pas le père biologique comme le procréateur, mais seulement comme celui qui ouvre le passage à l'enfant, sur lequel il n'a que peu de droit parce que ce dernier appartient au clan dont est issue la mère, la figure d'autorité étant l'oncle maternel.

Dans les sociétés matrilinéaires, la femme jouit d'un statut presque égal à celui de l'homme, même si, là comme ailleurs, demeure ce que Françoise Héritier (1981) appelle la «valence différentielle des sexes ». Autrement dit, même si les hommes dominent, les femmes y sont en position plus favorable, presque à égalité avec eux, au point que les autres systèmes, en voisins médisants, les accusent de leur être complètement soumis. De là serait née la légende des Amazones. Les sociétés matrilinéaires ont quasiment disparu alors qu'elles ont sans doute été le modèle dominant pendant une longue période de l'histoire de l'humanité, notamment aux débuts du Néolithique ${ }^{1}$. Ce n'est pas qu'elles étaient plus archaïques ou moins morales (comme le pensaient les premiers ethnologues), mais plutôt qu'elles étaient plus paisibles, plus pacifiques et plus égalitaires et donc plus vulnérables, étant moins en mesure de se défendre face aux sociétés patrilinéaires.

\section{Sexualité et fragilité de la famille hypermoderne}

Jusqu'à une période récente, la famille constituée d'hommes et de femmes de plusieurs générations, souvent élargie aux cousins et cousines, a été la base fonctionnelle de production des conditions matérielles d'existence, comme de reproduction de la vie, d'élevage des enfants et de transmission des valeurs propres à chaque société. Ce modèle a commencé à se transformer avec l'urbanisation et l'industrialisation, mais plus radicalement et plus récemment encore avec l'avènement progressif des sociétés 
modernes où l'État et les assurances privées prennent le relais des anciennes formes de solidarité assurées par la famille qui garantissaient jusque-là pour l'essentiel la protection du couple mère/enfant, mais aussi des vieillards, des malades et des indigents. À cela s'ajoute encore pour la période actuelle, l'impact des médias numériques qui diffusent de nouveaux modèles dont on a encore bien du mal à mesurer les effets tant ils sont récents, mais aussi parce que la culture, notamment les valeurs et les règles régissant la sexualité, évolue lentement, toujours moins vite que les transformations matérielles des sociétés.

Dans le melting pot où sont jetées toutes les cultures, les règles, habitudes et injonctions - qui jusque-là organisaient strictement la sexualité, le couple et la famille sont toutes brassées entre elles et fortement relativisées. Cette déstabilisation favorise l'hégémonie planétaire du modèle romantique occidental servi à l'envi par les industries culturelles. Les "grosses productions» (blockbusters), principalement nord-américains, alimentent et formatent les univers mentaux. Les rencontres sont présentées essentiellement jusqu'à la première scène d'amour, toujours incandescente et passionnée. Ils traitent bien plus rarement de la vie du couple moderne et moins encore de ses difficultés, ou à peine, pour préparer une rupture, suivie d'une nouvelle rencontre. Les couples se forment parce que les conjoints s'aiment, ils se séparent quand la vie sexuelle et sentimentale avec le partenaire ne les satisfait plus. La fidélité est une obligation absolue: pourquoi rester ensemble si on ne s'aime plus? Il n'y a que dans le cinéma français, notamment dans les films d'auteur, que l'on voit encore des conjoints se tromper allègrement sans que cela ne porte à conséquence. Pour les sociétés plus traditionnelles du reste de la planète, engagées dans une course à marche forcée vers l'hypermodernité, les telenovela à grand succès mettent en scène les crises familiales dramatiques que suscite la passion romantique en rupture avec les règles traditionnelles qui jusque-là organisaient les mariages arrangés (Rasse et Ghinéa, 2011).
Dans les sociétés traditionnelles et jusqu'au $\mathrm{XX}^{\mathrm{e}}$ siècle, à l'exception des sociétés matriarcales, les relations sexuelles n'étaient autorisées qu'après le mariage arrangé selon des contingences matrimoniales où la sexualité comptait pour peu, l'objectif premier étant de fonder ou de maintenir et de développer une unité de production familiale viable. Dans la société hypermoderne, l'attraction sexuelle est le moteur de la rencontre qui doit aboutir, si tout se passe bien, moins au mariage qu'à la constitution de couples, domaine par excellence d'épanouissement du bonheur promis par la société de consommation.

Grâce à la contraception, les jeunes peuvent faire l'expérience dès l'adolescence d'une sexualité «sans conséquence »; mais assez vite, le choix du partenaire est orienté par le projet de former un couple relativement stable. Le couple conjugal moderne repose sur la possibilité de choisir son conjoint par amour, selon une alchimie subtile combinant une sexualité épanouie et la possibilité de se projeter dans l'avenir avec un ou une partenaire pour lequel on ressent une certaine affinité. Fonder une famille demeure un horizon d'attente pour la plupart des jeunes en âge de se marier, l'idéal sublime, un refuge affectif, le dernier lieu de solidarité échappant à la monétarisation des échanges. Mais plus cet idéal est fort, et moins il convient aux petits arrangements avec la réalité de la vie domestique.

Les couples mariés, pacsés, concubins, se séparent parce qu'ils en ont la possibilité légale et matérielle mais, bien plus que cela, parce qu'ils ne s'aiment plus de l'amour passionnel qui animait leur rencontre et qu'ils n'y sont pas contraints par des contingences et des obligations qui jusque-là dépassaient leur propre existence et leur permettaient néanmoins de la sublimer.

La divortialité croissante témoigne, pour le moins, d'un malaise de la vie conjugale, de la fragilité du lien au regard de l'investissement et des attentes de la vie amoureuse, censée en constituer l'essence même. Les individus sont écartelés par des injonctions paradoxales. D'un côté, la famille, même réduite à sa plus simple expression, 
demeure un espace privilégié d'épanouissement et de bonheur et le dernier rempart contre la solitude physique. De l'autre, l'individu doit se réaliser coûte que coûte, cultiver sa personnalité. La modernité lui impose d'être lui-même. Passant sans cesse d'un monde à l'autre, obligé de changer de jeu, de rôle, de statut, d'identité, incertain sur ce qu'il est vraiment, il lui faut sans cesse se rassurer, vérifier ses capacités de séduction, être aimé... L'homo sexualis, commente Bauman (2004), toujours en quête d'identité, de réalisation de soi, inquiet « d’avoir oublié, raté, négligé, de n'avoir pas essayé, ni exploré quelque chose de capital; de ne pas s'être acquitté de certaines obligations vitales à son propre moi authentique», multiplie, ou rêve de multiplier, les expériences de rencontre, de découverte, de transgression.

Le corps est mis en évidence, dans un univers où la publicité multiplie les allusions sexuelles, les images sensuelles, où le désir est hypertrophié. En même temps, l'amour doit être absolu, ou pour le moins, la fidélité demeure une valeur forte, fondatrice du lien matrimonial, quand tout le reste se défait. Dans les blockbusters comme dans la vie, le conjoint pardonne rarement les expériences extraconjugales. Puisque la séparation est toujours possible, le couple exige une relation forte, dense et de fidélité absolue qui a bien du mal à se vivre au quotidien. L'insatisfaction conduisant au divorce, conclut François de Singly (2004), provient de ce que les partenaires ont trop idéalisé la "postmodernité conjugale» et qu'ils ne parviennent plus à jouer suffisamment le jeu, à participer à l'élaboration de compromis obligatoires (cf. Bastard, 2001).

Aujourd'hui, en France, 18 \% des enfants mineurs vivent avec deux adultes non mariés, $16 \%$ avec un seul adulte au sein d'une famille monoparentale et près de $10 \%$ vivent dans une famille recomposée (Festy, 2013). Moins d'une personne sur trois habite dans une famille composée du père, de la mère et des enfants. Et même dans ce cas, vivent-ils vraiment ensemble, si l'on observe que depuis le plus jeune âge chacun est irrémédiablement absorbé par une existence qui se construit ailleurs, à l'extérieur, ou au moyen d'écrans de plus en plus nombreux et sophistiqués? De fait, les gens continuent de se marier et fonder des familles, mais celles-ci durent peu - cinq à sept ans disent les statistiques. Les divorces concluent quatre mariages sur dix. Les pactes civils de solidarité (pacs) durent en moyenne deux ans et finissent une fois sur deux par un mariage et une fois sur deux par une séparation. Le nombre de familles monoparentales va croissant $(12,4 \%$ en $1990,21,2 \%$ en $2010^{2}$ ). Il faut encore prendre en considération l'allongement de la durée de vie qui fait que les parents peuvent passer la moitié de leur existence sans les enfants, à deux ou seuls après une séparation ou le décès de l'un d'entre eux. Autrement dit, la seule chose qui progresse réellement, c'est le nombre de célibataires, ou plutôt le temps durant lequel les gens sont célibataires.

Au final, tout atomisé qu'il soit, ce modèle contemporain, largement répandu et en progression partout dans le monde, bien qu'il en demeure d'autres, rejoint celui des sociétés matrilinéaires paisibles, car là aussi, il disjoint la sexualité de la filiation. Avec la généralisation de la contraception, le fait d'avoir des relations sexuelles pour assurer une descendance patrilinéaire légitime ne dure qu'un temps, de plus en plus court, d'autant plus court que l'espérance de vie progresse. Passée cette étape, les conjoints peuvent se séparer, former d'autres couples et pourquoi pas de même sexe. Les mères de famille monoparentale, notamment, peuvent bien continuer d'avoir la sexualité qu'elles veulent avec des amants visiteurs ou au sein de familles recomposées. Plus généralement, la question de la filiation, légitime ou non, n'est plus véritablement un problème; elle engage surtout de l'affection, l'héritage à transmettre est la plupart du temps davantage culturel que matériel. Les enfants issus des précédents mariages peuvent être formellement adoptés ou simplement élevés par les nouveaux conjoints, tandis que les progrès des techniques 
d'empreinte génétique peuvent rassurer définitivement les sujets inquiets sur leur paternité. Ajoutons encore que même s'il demeure une prévalence masculine, dans beau-

\section{N O T E S}

1. Robert Deliège (1996) évalue le nombre de sociétés matrilinéaires à $15 \%$ d'un échantillon de 565 sociétés connues (contre $44 \%$ de sociétés patrilinéaires et $41 \%$ de sociétés bilinéaires), mais elles n'existent probablement plus aujourd'hui qu'à l'état de traces.

\section{RÉ F É R E N C E S B I B L I O G R A P H I Q U E S}

BASTARD, B., «La séparation, mais le lien», Terrain, n 36, 2001, p. 5-16.

Bauman, Z., L'Amour liquide. De la fragilité des liens entre les hommes, Arles, Le Rouergue/Chambon, 2004.

Clastres, P., La Société contre l'État, Paris, Minuit, 2001.

Deliège, R., Anthropologie de la parenté, Paris, Armand Colin, 1996.

DE Singly, F., Sociologie de la famille contemporaine, Paris, Armand Colin, 2004

FeSty, P., «Le mariage et la famille en mouvement», Commentaire, $\mathrm{n}^{\circ} 142,2013$, p. 289-294.

Fox, R., Anthropologie de la parenté, une analyse de la consanguinité et des alliances, Paris, Gallimard, 1972.

HÉRITIER, F., Masculin/féminin, la pensée de la différence, Paris, Odile Jacob, 1996. coup de pays du monde les femmes n'ont jamais été aussi égales dans leurs relations avec les hommes, libres de leurs choix et de leur sexualité.

2. En France, selon l'Insee. Voir: <www.insee.fr/fr/themes/ tableau.asp?reg_id=0\&ref_id=amfd3>, consulté le 06/05/2014.

LÉvi-Strauss, C., "The Family», in Shapiro, H. L. (dir.), Man, Culture and Society, New York, Oxford University Press, 1956, p. 261-285. Traduction en langue française: «La famille», Annales de l'université d'Abidjan, série F 3, fasc. 3, Ethnosociologie, 1971, p. 5-29.

LÉVI-STRAUSs, C., Les Structures élémentaires de la parenté, Paris, Mouton, 1981.

MalinowsKi, B., La Vie sexuelle des sauvages du nord-ouest de la Mélanésie, Paris, Payot, 1930.

RASSE, P., La Rencontre des mondes: diversité culturelle et communication, Paris, Armand Colin, 2006.

RAsse, P. et GhineA, L., «Persistances et mutation des dernières sociétés rurales: le cas du pays Maramures», Terrain, ${ }^{\circ}$ 57, 2011, p. 114-127. 